\title{
Evaluación de la Estabilidad Oxidativa del Biodiesel de Jatropha (Jatropha curcas $L$.) mediante el uso de Antioxidantes Sintéticos y Biodiesel de Palma
}

\author{
Carlos A. Aguilar ${ }^{(1)}$, Kellys Rodríguez ${ }^{(1)}$, Susan C. González $^{(1)}$ y Luis A. Rios ${ }^{(2)}$ \\ (1) Grupo de Energías Alternativas y Biomasa GEAB, Universidad Popular del Cesar. Valledupar-Colombia \\ (e-mail: krodriguezescobar@gmail.com; caaguilart@gmail.com) \\ (2) Grupo Procesos Químicos Industriales, Universidad de Antioquia. Calle 70 № 52-21, Medellín-Colombia \\ (e-mail: lariospfa@gmail.com)
}

Recibido Jul. 24, 2014; Aceptado Oct. 6, 2014; Versión final recibida Dic. 5, 2014

\begin{abstract}
Resumen
El propósito de esta investigación fue la mejora de la estabilidad oxidativa del biodiesel de jatropha (Jatropha curcas L.) a través del uso de antioxidantes sintéticos comerciales y biodiesel de palma, el referente nacional en Colombia. Cada tipo de biodiesel se obtuvo por transesterificación básica con metanol y se utilizaron butil hidroxi tolueno, ascorbil palmitato y propil galato en diferentes concentraciones (250, 500, 750 y $1000 \mathrm{ppm}$ cada uno), como antioxidantes sintéticos. Se realizaron mezclas entre biodiesel de jatropha y biodiesel de palma como estrategia para obtener un biodiesel con mejor estabilidad oxidativa, la cual se evaluó siguiendo el estándar de la norma EN 14112 para tiempo de inducción. Los resultados mostraron que todos los antioxidantes evaluados ejercen un efecto positivo en la estabilidad oxidativa, siendo mejor al utilizar propil galato.
\end{abstract}

Palabras clave: biodiesel, Jatropha curcas L., transesterificación, estabilidad oxidativa, antioxidantes, propil galato

\section{Evaluation of Oxidative Stability of Jatropha (Jatropha curcas L.) Biodiesel by use of Synthetics Antioxidants and Palm Biodiesel}

\begin{abstract}
The purpose of this research was improving the oxidative stability of biodiesel from jatropha (Jatropha curcas $L$.) through the use of commercial synthetic antioxidants and biodiesel from palm, the Colombian national standard. Each type of biodiesel was obtained by basic transesterification with methanol, using butyl hydroxy toluene, ascorbyl palmitate and propyl gallate at different concentrations $(250,500,750$ and $1000 \mathrm{ppm}$ each), as synthetic antioxidants. Mixtures were prepared with biodiesel from jatropha and palm biodiesel as a strategy to get a better oxidative stability of biodiesel, which was evaluated following the standard EN 14112 for induction time. The results showed that all antioxidants tested have a positive effect on the oxidative stability, being better the use of propyl gallate.
\end{abstract}

Keywords: biodiesel, Jatropha curcas L., transesterification, oxidative stability, antioxidants, propyl gallate 


\section{INTRODUCCIÓN}

A través de los años los combustibles fósiles se han convertido en la principal fuente de energía en el mundo, tanto así que son considerados de necesidad primordial para que los países alcancen un alto grado de desarrollo económico. Esta dependencia, ha dado lugar que con el pasar del tiempo las reservas existentes de petróleo en el planeta estén agotándose por su constante explotación, trayendo consigo una de las consecuencias más anunciadas: el aumento en el precio del crudo (Jain et al., 2011a). Junto a lo anterior se suma el hecho de que la quema de combustibles fósiles da lugar al aumento de las concentraciones de gases de invernadero, convirtiéndose así en una problemática ambiental de interés general.

En el caso de combustibles alternativos para motores diésel, el biodiesel ha tenido gran auge, debido a que se obtiene de grasas y aceites naturales, y a que su uso disminuye las emisiones contaminantes con respecto al combustible fósil (Marticorena et al., 2010). Colombia, un país con gran vocación agrícola, posee gran diversidad de materias primas útiles para la producción de biodiesel, siendo el aceite de palma la principal fuente de aceite para la producción de biodiesel a nivel nacional (Zuleta 2012, a). Sin embargo, la naturaleza de los metilésteres que conforman el biodiesel hace que presente algunos inconvenientes. Una de las principales dificultades es la oxidación de biodiesel, la cual es motivo de preocupación en el sector automotriz, debido a que los productos de oxidación podrían ocasionar corrosión sobre los materiales que estén en contacto con ellos. Además, la oxidación del biodiesel en su etapa de almacenamiento, puede originar pérdida de su calidad antes de ser usado.

El aceite de semilla de jatropha (Jatropha curcas) es visto en Colombia como una alternativa para la producción de biodiesel que no compite con las aplicaciones que el aceite de palma tiene en el sector agroalimentario. Por otra parte, la composición química del aceite de jatropha le provee propiedades fisicoquímicas, como buenas propiedades de flujo en frio, en cierto grado mejor que las de otros aceites (Sarin et al., 2007). El biodiesel de aceite de jatropha obtiene algunas propiedades fisicoquímicas del aceite que lo origina: este biodiesel tiene buenas propiedades de flujo en frio ya que contiene cerca del $80 \%$ de ácidos grasos insaturados, donde los metilésteres de los ácidos oleico y linoleico, se encuentran en mayor cantidad y por tal razón la estabilidad oxidativa y el punto de nube de este biodiesel son bajos, a diferencia del biodiesel de aceite de palma cuya composición en ácidos grasos saturados es alta, confiriéndole una buena estabilidad oxidativa pero malas propiedades de flujo en frío (Zuleta et al., 2012).

La mezcla de biodiéseles de diferentes aceites es una técnica que ha sido estudiada para mejorar las propiedades de este biocombustible. Esta técnica se basa en que existen algunos biodiéseles, que por su composición, presentan valores adecuados en algunos parámetros pero no en otros. Por citar un ejemplo, el biodiesel de aceite de palma posee buenas propiedades de estabilidad oxidativa pero malas propiedades de flujo en frío, mientras que el biodiesel de aceite de soya presenta características opuestas; una mezcla entre ellos en determinada proporción, podría mejorar las propiedades de flujo en frío y la estabilidad oxidativa del producto final. El objetivo principal de esta investigación se centró en evaluar diversas concentraciones de antioxidantes sintéticos y mezclas binarias con biodiesel de aceite de palma como alternativas para mejorar la estabilidad oxidativa del biodiesel de aceite de jatropha y poder así realizar un aporte a las investigaciones realizadas en cuanto a calidad de este biodiesel.

\section{MATERIALES Y MÉTODOS}

\section{Preparación y caracterización de los biodiéseles}

El procedimiento experimental inició con la determinación del índice de acidez del aceite refinado, blanqueado y desodorizado de palma y del aceite crudo de jatropha según la norma NTC 218 (ICONTEC 1999). La producción del biodiesel de aceite de palma se realizó por transesterificación con metanol (relación molar metanol aceite 9:1) utilizando $\mathrm{KOH}$ como catalizador $(0,7 \% \mathrm{p} / \mathrm{p}$ en base al aceite). Esta reacción se llevó a cabo en un reactor para biodiesel construido en acero inoxidable con condensador de reflujo, control de temperatura y un sistema de agitación a $60{ }^{\circ} \mathrm{C}$, presión atmosférica, y un tiempo de reacción de 1,5 horas. Transcurrida la reacción, se dejó reposar en el reactor durante 24 horas para separar el biodiésel crudo de la glicerina.

El biodiésel de aceite de jatropha se obtuvo por esterificación ácida (concentración de catalizador al $1 \%$ $\mathrm{H}_{2} \mathrm{SO}_{4}, 26 \%$ de metanol, temperatura de reacción $60^{\circ} \mathrm{C}$, presión atmosférica, y tiempo de reacción de 2 horas) seguida de transesterificación básica en dos etapas (relación molar metanol aceite de 9:1 y 7:1) con $\mathrm{KOH}$ como catalizador $(0,7 \% \mathrm{p} / \mathrm{p}$ en base al aceite) . 
A los biodiéseles obtenidos en los procedimientos descritos, se les realizaron pruebas de índice de acidez, contenido de metiléster y estabilidad oxidativa. El índice de acidez se evaluó por titulación manual y con la ayuda de un titulador automático 848 Titrino Plus (Metrohm). El contenido de metiléster se determinó mediante cromatografía gaseosa siguiendo la norma europea EN 14103 (CEN 2003, a). Se utilizó un equipo de cromatografía gaseosa (Agilent 7890A) con una columna capilar (Agilent J\&W HP-Innowax), un detector de ionización de llama (FID), y tetradecano $\left(\mathrm{C}_{14} \mathrm{H}_{30}\right)$ como estándar interno. La estabilidad oxidativa se determinó con un equipo 873 Biodiésel Rancimat (Metrohm), siguiendo la norma EN 14112.

\section{Evaluación de diferentes antioxidantes}

Se evaluó el efecto de la adición de diferentes concentraciones de los antioxidantes butil hidroxi tolueno, ascorbil palmitato y propil galato $(250,500,750$ y $1000 \mathrm{ppm})$, sobre la estabilidad oxidativa del biodiesel de aceite de jatropha, a una temperatura de $110^{\circ} \mathrm{C}$ siguiendo la norma EN 14112 (CEN 2003, b).

\section{Evaluación de la cinética de reacción de oxidación}

Unas vez obtenidos los resultados de la estabilidad oxidativa de los diferentes mezclas, se realizaron los cálculos para determinar la concentración crítica y la vida útil utilizando las ecuaciones (1), (2), (3), (4) que relacionan de manera logarítmica el tiempo de inducción, las concentraciones crítica e inicial y la temperatura a la que se realizaron las pruebas. La ecuación (5) se utilizó para establecer el tipo de reacción, relacionando la energía de activación, la temperatura y la constante de los gases ideales, de acuerdo con lo reportado por Chen y Luo (2011) y Xin y otros (2009).

$$
\begin{aligned}
& \frac{d c}{c}=-k d t \\
& \ln C_{0}-\ln C_{c r}=k t_{i}-k t_{i 0} \\
& t_{i}=\frac{1}{k} \ln \frac{C_{0}}{C_{c r}}+t_{i 0} \\
& \ln C_{0}=k\left(t_{i}-t_{i 0}\right)+\ln C_{c r}
\end{aligned}
$$

\section{Evaluación de las Mezclas de los biodiesel palma/jatropha}

Se realizaron mezclas binarias de los biodiéseles de palma y jatropha en proporciones P90/J10, P80/J20, P70/J30, P60/J40, P50/J50, P40/J60, P30/J70, P20/J80, P10/J90 (peso/peso), (el primer valor, P, equivale a la cantidad de biodiesel de palma en la mezcla, y el segundo valor $\mathrm{J}$ equivale al de biodiesel de jatropha).

\section{RESULTADOS Y DISCUSION}

\section{Producción de los biodiéseles y su caracterización}

En la Tabla 1 se presentan los valores requeridos según la resolución 182087 de 2007 del Ministerio de Ambiente, Vivienda y Desarrollo Territorial y Ministerio de Minas y Energía, (Minenergia, 2007). Según los valores obtenidos, el contenido de metilésteres sobrepasa los valores de la resolución nacional, lo que indica alta conversión en la reacción de transesterificación. El índice de acidez tanto para el biodiesel de aceite de palma, como para el biodiesel de aceite de jatropha presenta valores aceptables, siendo un poco más alto para el biodiesel de aceite de jatropha. La Tabla 2 muestra la composición de metilésteres de los biodiéseles de palma y jatropha. Como se puede observar, el biodiesel aceite de palma tiene aproximadamente el mismo contenido de metil palmitato y metil oleato, lo que le confiere una relación homogénea de metilésteres insaturados/saturados. El biodiesel de jatropha tiene el un alto porcentaje de metilésteres de ácido palmítico, pero tiene el mayor contenido de metiléster de ácido linoléico.

Tabla 1: Resultados de caracterización de los biodiéseles de palma y jatropha.

\begin{tabular}{|l|c|c|c|}
\hline \multirow{2}{*}{ Prueba } & \multicolumn{2}{|c|}{ Biodiésel } & \multirow{2}{*}{$\begin{array}{c}\text { Res. } 182087 \\
\text { de } 2007\end{array}$} \\
\cline { 2 - 3 } & Palma & Jatropha & $<0,5$ \\
\hline Índice de acidez (mg KOH/g muestra) & 0,32 & 0,51 & $>96,5$ \\
\hline Contenido de metiléster (\%) & 97,77 & 98,20 & $<$ \\
\hline
\end{tabular}




\section{Eficiencia de los antioxidantes en la mejora de la estabilidad oxidativa del biodiesel de aceite de jatropha}

Se evaluó la estabilidad oxidativa del biodiesel de jatropha puro y en mezcla con antioxidantes, utilizando un equipo 873 Biodiésel Rancimat (Metrohm), y bajo los parámetros establecidos en la norma EN 14112. La Fig. 1 presenta los resultados del tiempo de inducción para las diferentes mezclas de biodiesel de aceite de jatropha con los antioxidantes evaluados. Como se puede observar, el biodiesel de aceite de jatropha presenta un tiempo de inducción de 1.11 horas, el cual está por debajo de lo establecido en la normatividad nacional (6 horas). Este valor en el tiempo de inducción, el cual indica una mala estabilidad oxidativa, esta relacionado con el lato contenido de metilésteres insaturados, tal como lo presentan otoros autores como Jain (2011, b), Freire y otros. (2012), Sarin y otros (2010) y Chaithongdee, Chutmanop y Srinophakun (2010). De los tres antioxidantes sintéticos evaluados en el biodiesel de aceite de jatropha, solo el propil galato en concentración de 500 ppm son aumentan el tiempo de inducción del biodiesel. El butil hidroxi tolueno y el propil galato presentan valores estadísticamente similares de tiempo de inducción a todas las concentraciones evaluadas (Fig. 1).

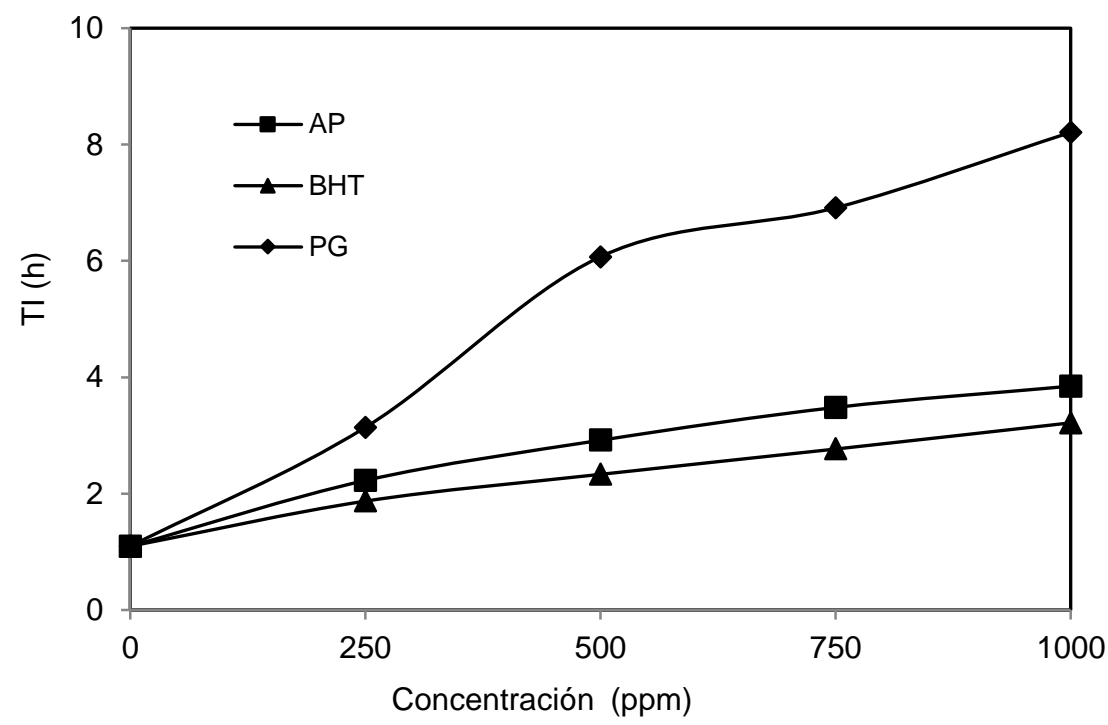

Fig. 1: Estabilidad oxidativa del biodiesel de aceite de jatropha en mezcla con ascorbil palmitato, butil hidroxi tolueno y propil galato a $110{ }^{\circ} \mathrm{C}$ (Intervalos de mínima diferencia significativa al 95\% de confianza)

Tabla 2: Composición de metilésteres de los biodiéseles de palma y jatropha

\begin{tabular}{|l|c|c|c|}
\hline \multirow{2}{*}{ Metiléster } & \multirow{2}{*}{$\mathcal{N}^{\circ}$ enlaces } & \multicolumn{2}{|c|}{ Composición (\%) } \\
\cline { 3 - 4 } & & Palma & Jatropha \\
\hline Laurato & $12: 00$ & 0,218 & 0,000 \\
\hline Miristato & $14: 00$ & 0,923 & 0,090 \\
\hline Palmitato & $16: 00$ & 42,551 & 12,993 \\
\hline Palmitoleato & $16: 01$ & 0,156 & 0,700 \\
\hline Estearato & $18: 00$ & 4,577 & 7,363 \\
\hline Oleato & $18: 01$ & 40,776 & 42,723 \\
\hline Linoleato & $18: 02$ & 10,056 & 35,609 \\
\hline Linolenato & $18: 03$ & 0,192 & 0,203 \\
\hline Eicosanato & $20: 01$ & 0,387 & 0,225 \\
\hline Eicosenato & $20: 01$ & 0,160 & 0,090 \\
\hline MES, metilésteres saturados & & 48,269 & 20,446 \\
\hline MEMI, metilésteres monoinsaturados & & 41,479 & 43,738 \\
\hline MEPI: metilésteres poliinsaturados & & 10,248 & 35,812 \\
\hline
\end{tabular}




\section{Evaluación de la cinética de reacción de oxidación}

Las concentraciones de propil galato y las temperaturas evaluadas fueron $0,250,500,700$ y $1000 \mathrm{ppm}$, a temperaturas de $90,100,110$ y $120^{\circ} \mathrm{C}$. Se observa en la fig. 2 que a mayor temperatura, el tiempo de inducción de las diferentes concentraciones de propil galato adicionadas al biodiesel de jatropha, disminuye. Si bien a $90^{\circ} \mathrm{C}$ y a $100^{\circ} \mathrm{C}$ las concentraciones de propil galato originan diferencias estadísticas significativas en el tiempo de inducción, a 110 y a $120^{\circ} \mathrm{C}$ los tiempos de inducción de las concentraciones evaluadas son estadísticamente iguales. Estudios han demostrado el efecto que ejerce la temperatura sobre la estabilidad oxidativa de diferentes tipos de biodiesel, afirmando así, que el tiempo de inducción obtenido a través del método Rancimat a una concentración de antioxidante particular (Pullen y Saeed, 2012), es inversamente proporcional al cambio de temperatura de análisis (Chen y Luo, 2011; Xin et al., 2009).

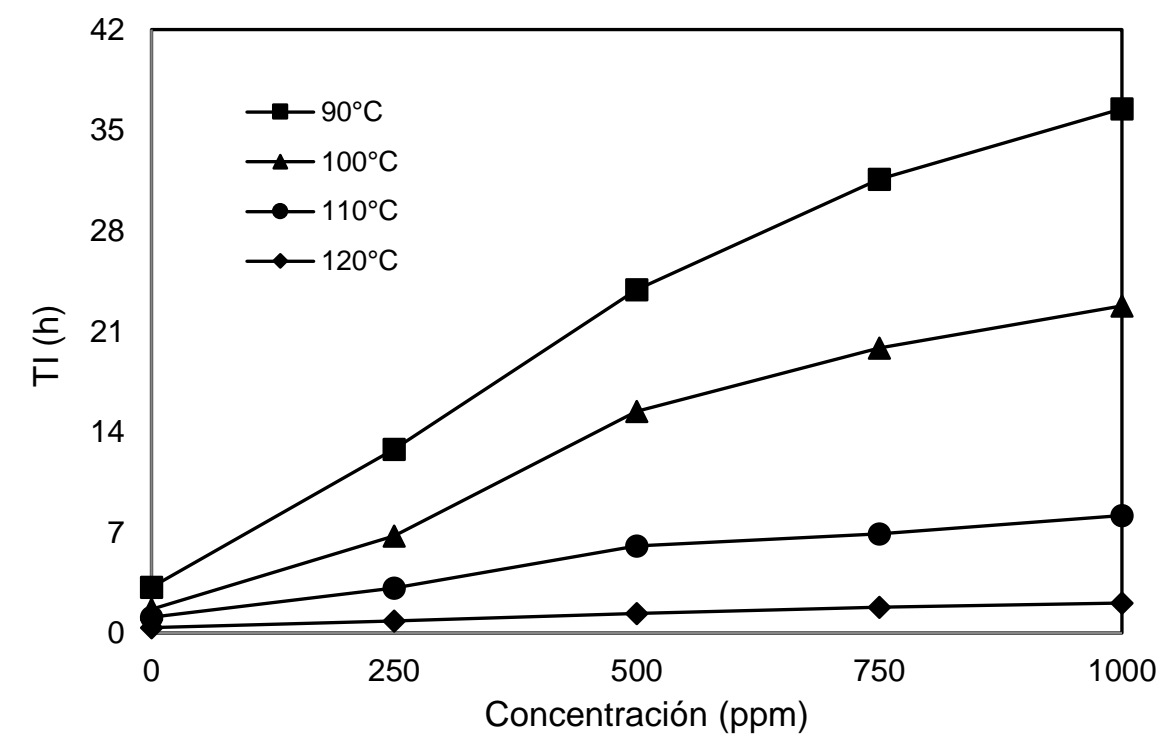

Fig. 2: Influencia de la concentración de propil galato en la estabilidad oxidativa del biodiesel de aceite de jatropha (Intervalos de mínima diferencia significativa al $95 \%$ de confianza)

La Fig. 3 se obtuvo a fin de ajustar los datos en líneas rectas para adoptar el modelo de la ecuación de velocidad de primer orden (1) (Xin et al., 2009). Las líneas muestran un alto grado de correlación tal como se aprecia en la Tabla 3, en la que todos los coeficientes superan 0,98 . Por lo tanto, el cambio que presenta el tiempo de inducción respecto a la concentración de propil galato puede determinarse mediante la pendiente de la línea recta utilizando la ecuación (4), ya que es igual a la constante de velocidad de consumo de propil galato $(k)$.

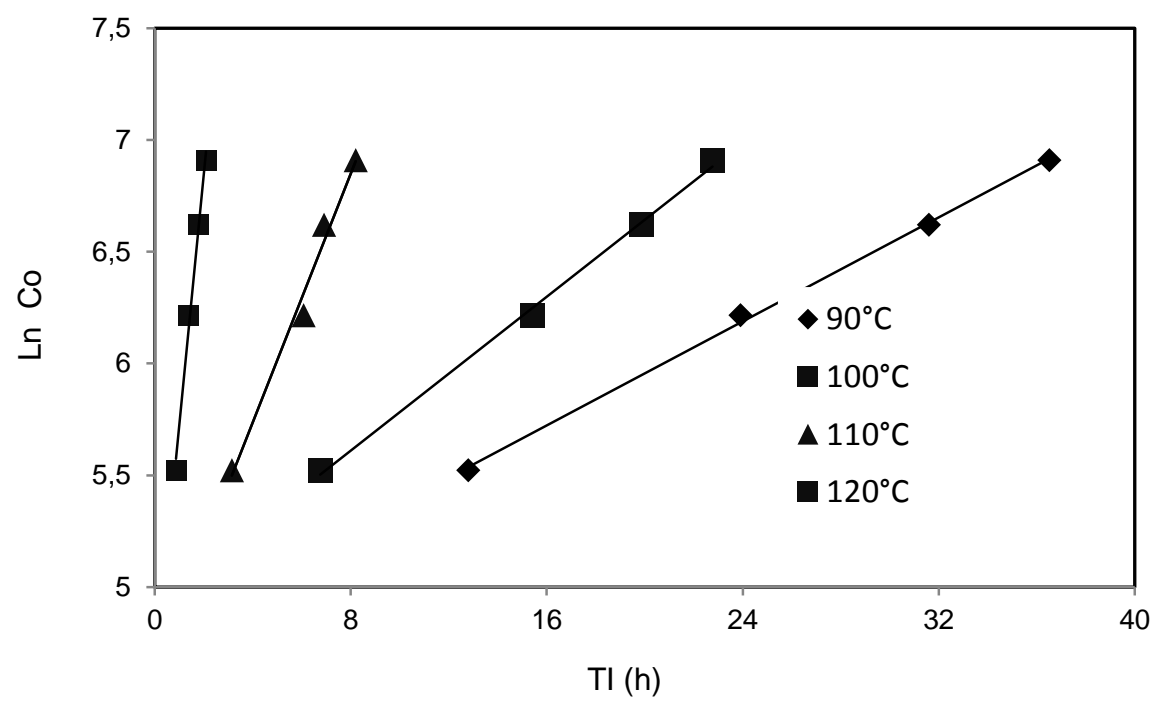

Fig. 3: Dependencia del tiempo de inducción con el logaritmo (In) de la concentración de propil galato a $90^{\circ} \mathrm{C}, 100^{\circ} \mathrm{C}, 110^{\circ} \mathrm{C}$ y $120^{\circ} \mathrm{C}$ 
En la Tabla 3 se aprecia que $k$ es directamente proporcional al aumento de la temperatura, siendo muy superior a $120^{\circ} \mathrm{C}$. La concentración crítica disminuye conforme el tiempo de oxidación y la temperatura aumentan. El consumo de propil galato está directamente relacionado con la temperatura.

La concentraciones críticas $C_{c r}$ a diferentes temperaturas se obtuvieron aplicando antilogaritmo a la expresión $\mathrm{InC}_{\mathrm{cr}}$ de las ecuaciones de la línea recta halladas a partir de la Fig. 5. Estas concentraciones estuvieron en un rango entre 137,6 - 101,8 ppm con un valor promedio de 115,5 ppm.

Tabla 3: Constante de reacción $(k)$ para el consumo de propil galato a varias temperaturas y coeficiente de correlación.

\begin{tabular}{|c|c|c|c|}
\hline Temperatura $\left({ }^{\circ} \mathrm{C}\right)$ & $K\left(h^{-1}\right)$ & $C_{\text {cr }}(\mathrm{ppm})$ & Coeficiente de correlación $R^{2}$ \\
\hline 90 & 0.0582 & 120.6 & 0.9987 \\
\hline 100 & 0.0860 & 137.6 & 0.9978 \\
\hline 110 & 0.2780 & 102.1 & 0.9856 \\
\hline 120 & 1.1066 & 101.8 & 0.9890 \\
\hline
\end{tabular}

Por otro lado, al graficar los valores del In $k$ que satisfacen la ecuación de Arrhenius contra $\mathrm{T}^{-1}$ para el propil galato (Fig. 4), se obtuvo la ecuación de la línea recta (6) tomando como base la ecuación (5):

$\operatorname{lnk}=-\frac{E_{a}}{R T}+B$

$y=-14.197 x+35.973$

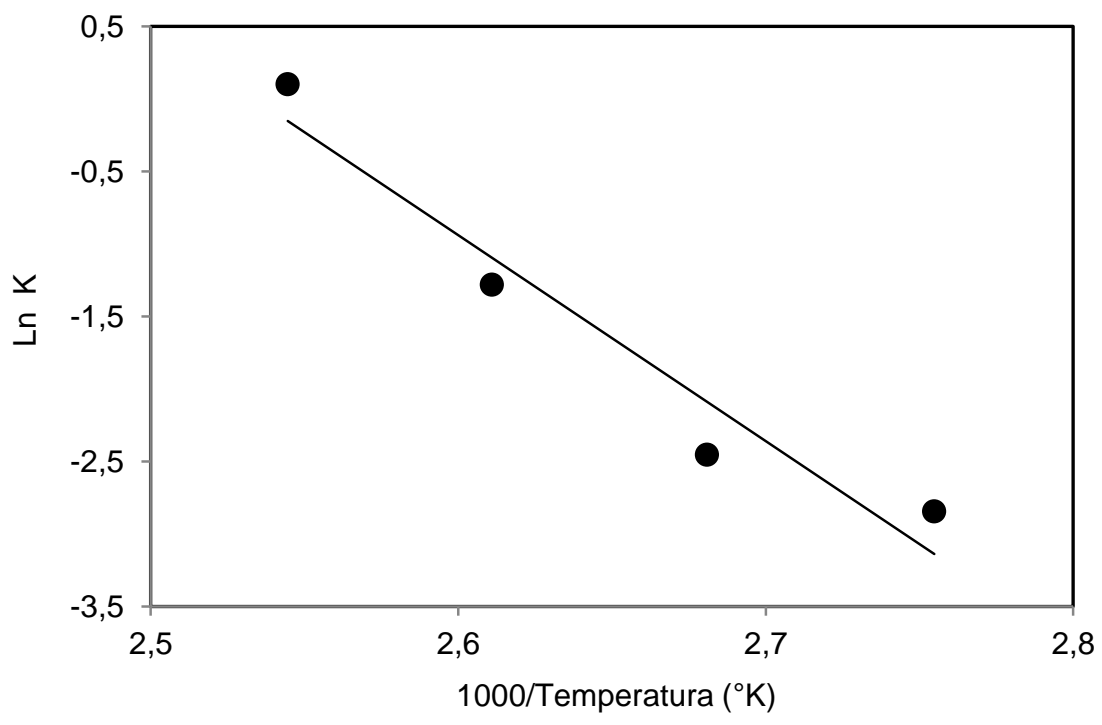

Fig. 4: Dependencia de k del propil galato con la temperatura.

Ya que (6) tiene la forma de la ecuación de la línea recta, al asumir $E_{a} / R$ como la pendiente, y despejando la expresión de la ecuación (5), se tiene:

$\operatorname{Ln} k=-\frac{1000}{R T} E_{a}+B$

$E_{a}=14.197 \times 8.314 \frac{\mathrm{kJ}}{\mathrm{mol}}$

$E_{a}=118,03 \frac{\mathrm{kJ}}{\mathrm{mol}}$ 
Al representar la energía de activación, el grado de sensibilidad a la temperatura y al comparar con los resultados de $E_{a}$ obtenidos para el consumo de propil galato $97,02 \mathrm{~kJ} / \mathrm{mol}$ (Xin et al., 2009) y Ethanox $4760 \mathrm{E} 85,68 \mathrm{~kJ} / \mathrm{mol}$ (Chen y Luo, 2011), se puede afirmar que el propil galato adicionado al biodiesel de jatropha es poco sensible a la temperatura.

Recientemente Xin et al (2009) y Chen y Luo (2011) demostraron la relación lineal entre el logaritmo natural del tiempo de inducción y la temperatura del biodiesel de aceite de girasol y el biodiesel obtenido de ácidos grasos libres a varias concentraciones, sus investigaciones indican que el mecanismo de consumo de antioxidantes sintéticos no cambia a temperatura ambiente, y por lo tanto las líneas rectas de la Fig. 5 pueden extrapolarse a bajas temperaturas y encontrar el tiempo de inducción a temperatura ambiente bajo condiciones reales de almacenamiento. Así, la Fig. 5 muestra una relación lineal entre el tiempo de inducción y la temperatura del biodiesel de aceite de jatropha en mezcla con propil galato en concentraciones de 250, 500, 750 y 1000 ppm.

La Fig. 6 muestra los resultados del tiempo de inducción obtenidos bajo el método de extrapolación. De acuerdo con los resultados, adicionando $750 \mathrm{ppm}$ de propil galato al biodiesel de aceite de jatropha se pueden garantizar 2,4 años de almacenamiento a $25^{\circ} \mathrm{C}$ sin que sufra degradación alguna, tiempo que puede ser considerado como un intervalo más realista para la vida comercial del biodiesel según algunos autores (Xin et al., 2009).

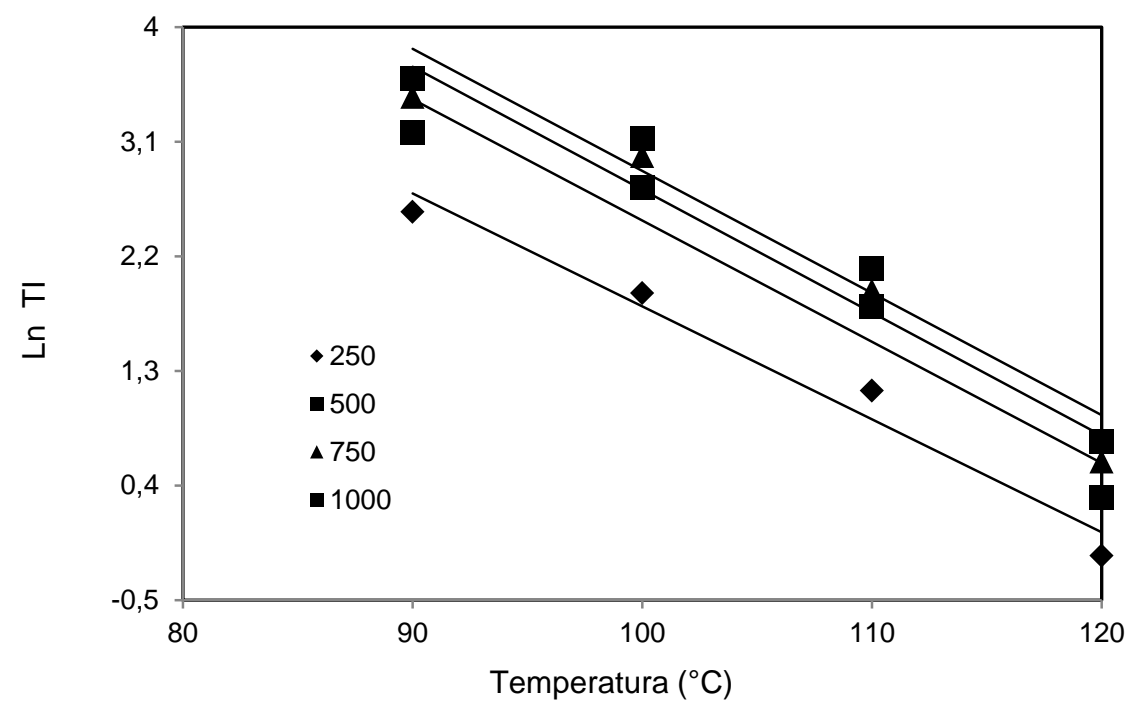

Fig. 5: Logaritmo del tiempo de inducción en función de la temperatura.

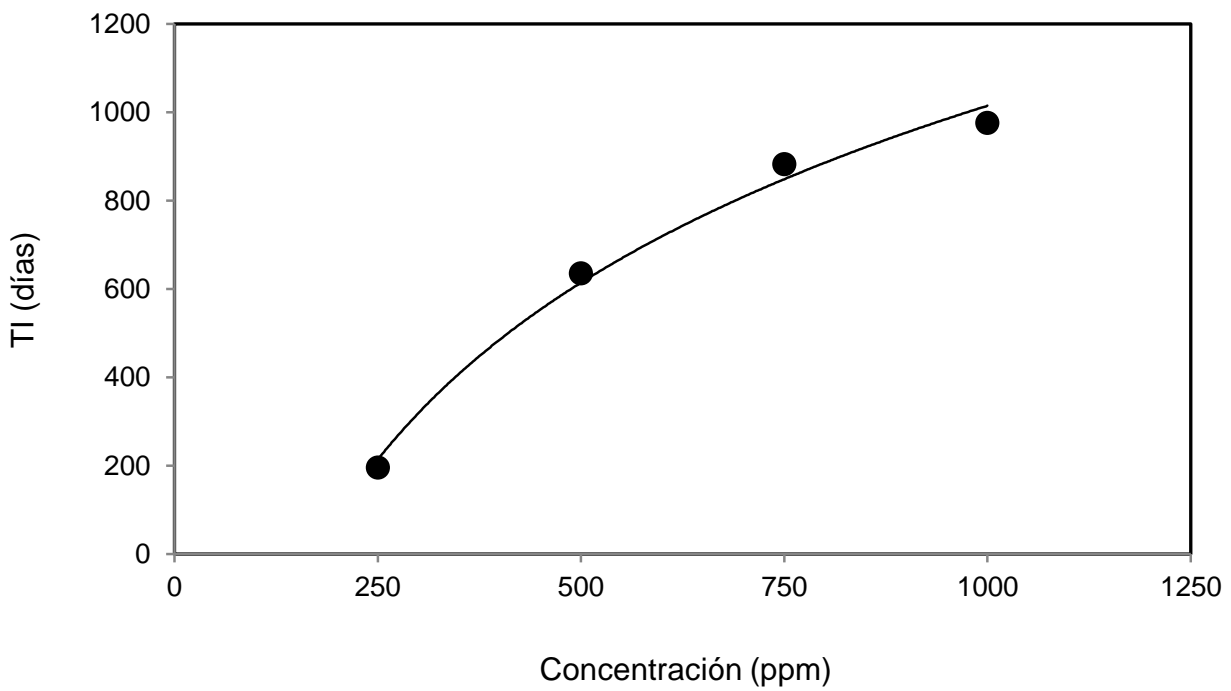

Fig. 6: Relación del tiempo de inducción (días) con la concentración de PG a $25^{\circ} \mathrm{C}$. 


\section{Evaluación de la estabilidad oxidativa del biodiesel}

Como alternativa para mejorar la estabilidad oxidativa del biodiesel de aceite de jatropha, se evaluó la adición de biodiesel de aceite de palma el cual contiene una elevada estabilidad oxidativa. Este procedimiento esta reportado para otros tipos de biodiéseles, pero no para los biodiéseles aquí evaluados (Moreira, 2013). El biodiesel de aceite de palma presenta un tiempo de inducción de 15,85 horas, valor similar al reportado por otros autores (Hincapié y otros, 2010). Esta alta estabilidad oxidativa está asociada a su alto contenido de metilésteres saturados (48,269\%, Tabla 2). Por otra parte, el biodiesel de jatropha presenta un contenido mayor en metilésteres insaturados (Rizqon, Prawoto y Bambang, 2012), induciendo a que el biodiesel sea menos estable y se degrade más rápidamente que el biodiesel de palma (Sierra, Quiñones y Rodriguez, 2010). El biodiesel de jatropha tuvo un tiempo de inducción de 1,11 horas, valor muy por debajo del requerido por la norma nacional que regula la calidad del biodiesel.

Los resultados de la Fig. 7 se pueden explicar teniendo en cuenta los perfiles de metilésteres de las diferentes mezclas generadas. Se utilizaron los datos obtenidos por cromatografía de gases para construir la Tabla 4, la cual muestra los perfiles de metilésteres correspondiente a cada una de las mezclas evaluadas. Se observa que de las mezclas evaluadas, sólo la mezcla con un $90 \%$ de biodiesel de aceite de palma y un $10 \%$ de biodiesel de aceite de jatropha (P90/J10) supera el tiempo de inducción de 6 horas establecido por la norma, a causa de que el $90 \%$ de la mezcla corresponde a biodiesel de palma, provocando a su vez la disminución del porcentaje de metilésteres poliinsaturados, en (12.804\%) en comparación con el alto contenido de los mismos en el biodiesel de jatropha puro. Además, se aprecia como las mezclas P90/J10 y P10/J90 son las que originan mayor y menor tiempo de inducción $(7,58 \mathrm{~h}$ y $1,21 \mathrm{~h}$ ), al igual se puede apreciar como el contenido de metilésteres poliinsaturados es mucho menor en la mezcla P90/J10 (12.804\%) que en J90/P10 (33.255\%).

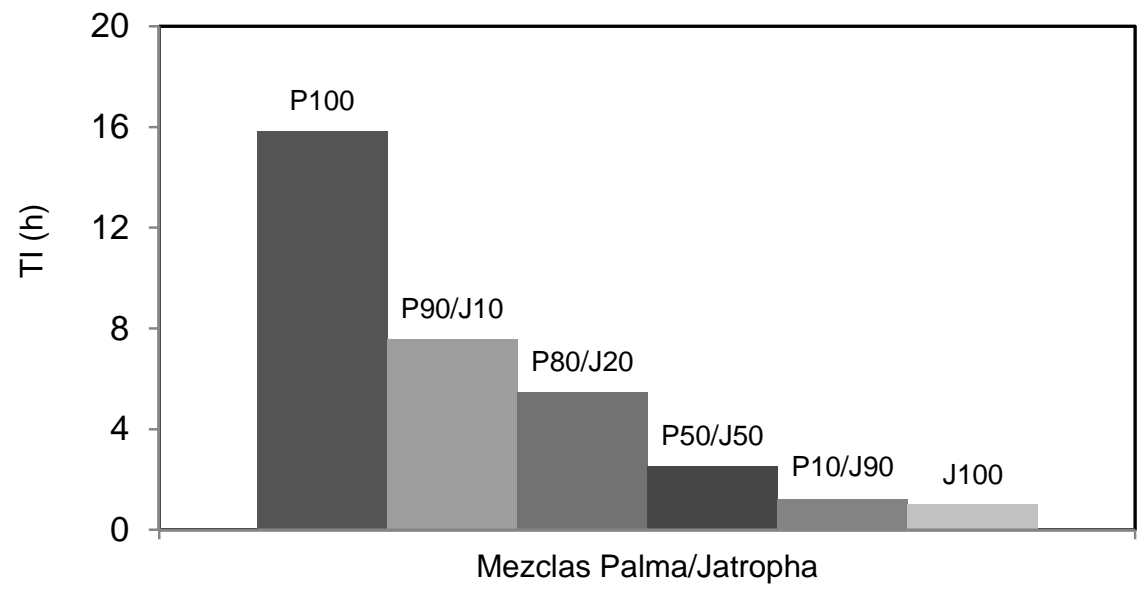

Fig. 7: Tiempo de inducción de los biodiéseles y sus mezclas (intervalos de mínima diferencia significativa al $95 \%$ )

Tabla 4: Composición de metilésteres (\%) de las mezclas evaluadas

\begin{tabular}{|c|c|c|c|c|c|c|c|c|c|}
\hline \multirow{2}{*}{ Metiléster } & \multicolumn{9}{|c|}{ Mezcla } \\
\hline & P90/J10 & P80/J20 & P70/J30 & $\mathrm{P} 60 / \mathrm{J} 40$ & $\mathrm{P} 50 / \mathrm{J} 50$ & P40/J60 & P30/J70 & P20/J80 & P10/J90 \\
\hline Laurato & 0.196 & 0.174 & 0.152 & 0.130 & 0.109 & 0.087 & 0.065 & 0.043 & 0.021 \\
\hline Miristato & 0.839 & 0.756 & 0.673 & 0.589 & 0.506 & 0.423 & 0.339 & 0.256 & 0.173 \\
\hline Palmitato & 39.595 & 36.639 & 33.683 & 30.727 & 27.772 & 24.816 & 21.860 & 18.904 & 15.948 \\
\hline Palmitoleato & 0.210 & 0.264 & 0.319 & 0.373 & 0.428 & 0.482 & 0.536 & 0.591 & 0.645 \\
\hline Estearato & 4.855 & 5.134 & 5.412 & 5.691 & 5.970 & 6.248 & 6.527 & 6.805 & 7.084 \\
\hline Oleato & 40.970 & 41.165 & 41.360 & 41.554 & 41.749 & 41.944 & 42.138 & 42.333 & 42.528 \\
\hline Linoleato & 12.611 & 15.166 & 17.721 & 20.277 & 22.832 & 25.387 & 27.943 & 30.498 & 33.053 \\
\hline Linolenato & 0.193 & 0.194 & 0.195 & 0.196 & 0.197 & 0.198 & 0.199 & 0.200 & 0.201 \\
\hline Eicosanato & 0.370 & 0.354 & 0.338 & 0.322 & 0.306 & 0.289 & 0.273 & 0.257 & 0.241 \\
\hline Eicosenato & 0.153 & 0.146 & 0.139 & 0.132 & 0.125 & 0.118 & 0.111 & 0.104 & 0.097 \\
\hline MES & 45.486 & 42.704 & 39.922 & 37.139 & 34.357 & 31.575 & 28.792 & 26.010 & 23.228 \\
\hline MEMI & 41.704 & 41.930 & 42.156 & 42.382 & 42.608 & 42.834 & 43.060 & 43.286 & 43.512 \\
\hline MEPI & 12.804 & 15.360 & 17.917 & 20.473 & 23.030 & 25.586 & 28.142 & 30.699 & 33.255 \\
\hline
\end{tabular}




\title{
CONCLUSIONES
}

El biodiesel de aceite de jatropha es un biodiesel que por sus propiedades fisicoquímicas presenta un gran potencial para la producción de biodiesel en Colombia, siendo su estabilidad oxidativa la principal debilidad para la aplicación industrial de este aceite. En este trabajo se presentaron dos alternativas para generar soluciones a este problema. La primera alternativa es la adición de antioxidantes, la cual arrojo como resultado que con la adición de propil galato a 500 ppm aumento el tiempo de inducción a 6,07 horas, alcanzando de esta manera los requerimientos nacionales para este parámetro. De los demás antioxidantes evaluados, ningún otro presentó una mejora significativa en el tiempo de inducción. En general el orden en el efecto que ejercieron los antioxidantes sobre el tiempo de inducción fue propil galato>ascorbil palmitato>butil hidroxi tolueno. Por otro lado este estudio demostró mediante un análisis realizado a partir de la cinética de reacción que el propil galato es moderadamente sensible a la temperatura, y que se deben tener condiciones de almacenamientos moderadas para que su poder antioxidante no se ve afectado por este factor.

\section{AGRADECIMIENTOS}

Los autores agradecen en primera instancia a la Universidad Popular del Cesar (Valledupar, Colombia) por el financiamiento de este trabajo y al grupo Procesos Químicos Industriales de la Universidad de Antioquia (Medellín, Colombia) por el apoyo en infraestructura, conocimientos y servicios técnicos brindados.

\section{NOTACIÓN}

\author{
$C_{0}$ : Concentración inicial de antioxidante \\ $C_{C r}$ Concentración crítica de antioxidante \\ $K \quad$ Constante de velocidad de consumo de antioxidante \\ $t_{i 0} \quad$ Tiempo de inducción sin antioxidante \\ $t_{i} \quad$ Tiempo de inducción con antioxidante \\ $R \quad$ Constante de gases ideales \\ $E_{a} \quad$ Energía de activación \\ B Constante que intercepta la línea recta con el eje vertical
}

\section{REFERENCIAS}

CEN, EN 14103. Productos derivados de aceites y grasas. Ésteres metílicos de ácidos grasos (FAME). Determinación de los contenidos de éster y de éster metílico de ácido linoleico, 1 - 14, España (2003).

CEN, EN 14112. 2003. Determinación de la estabilidad a la oxidación en biodiésel, 1 - 18, España (2003).

Chaithongdee, D., Chutmanop, J. y Srinophakun, P. Effect of antioxidants and additives on the oxidation stability of jatropha biodiesel. Kasetsart Journal (Natural Science): 44(2), $243-250$ (2010).

Chen, Y.-H. y Luo, Y.-M. Oxidation stability of biodiesel derived from free fatty acids associated with kinetics of antioxidants. Fuel Processing Technology: 92(7), 1387-1393 (2011).

Freire, L., y otros autores. Influence of the synthesis process on the properties of flow and oxidative stability of biodiesel from Jatropha curcas biodiesel. Fuel: 94(2), 313 - 316 (2012).

Hincapié, G., Ocampo, D., Restrepo, G. y Marín, J. Fotocatalisis heterogenea y foto - fenton aplicadas al tratamiento de aguas de lavado de la producción de biodiesel. Información Tecnológia: 22(2), 33 - 42 (2011)

Jain, S. y Sharma, M. Oxidation stability of blends of jatropha biodiesel with diesel. Fuel: 90(10), $3014-$ 3020 (2011a).

Jain, S. y Sharma, M. Optimization of long-term storage stability of Jatropha curcas biodiesel using antioxidants by means of response surface methodology. Biomass and Bioenergy: 35(9), 4008 - 4014 (2011b).

Marticorena, A., B, Mandagarán. y E, Campanella, Análisis del impacto ambiental de la recuperación de metanol en la producción de biodiésel usando el algoritmo de reducción de desechos WAR. Información Tecnológica: 21(1), 23 - 30 (2010). 
Minenergia y Medio ambiente., Resolución No. 180782 de mayo 30 de 2007, Por la cual se modifican los criterios de calidad de los biocombustibles para su uso 90 en motores diesel como componente de la mezcla con el combustible diesel de origen fósil en procesos de combustión. 2007

Moreira, E. Principales características de las materias primas utilizadas en la producción de biodiesel: la influencia del contenido y la concentración de los ácidos grasos. Ingenium, Facultad de Ingeniería: 25(3), 53 $-61(2013)$

NTC 218. Grasas y aceites vegetales y animales. Determinación del índice de acidez y de la acidez, $1-14$, Colombia (2011).

Pullen, J. y Saeed, K. An overview of biodiesel oxidation stability. Renewable and Sustainable Energy Reviews: 16(8), $5924-5950$ (2012).

Rizqon, F., Prawoto y Bambang, S. Predicting fuel properties of partially hydrogenated jatropha methyl esters used for biodiesel formulation to meet the fuel specification of automobile and engine manufacturers. Kasetsart Journal (Natural Science): 46(4), 629 - 637 (2012).

Sarin, R.; Sharma, S. Sinharay y R. Malhotra. Jatropha-Palm biodiesel blends: An optimum mix for Asia. Fuel 86, 1365-1371 (2007)

Sarin, R., y otros. Natural and synthetic antioxidants: Influence on the oxidative stability of biodiesel synthesized from non - edible oil. Energy: 35(12), 4645 - 4648 (2010).

Sierra, M., Quiñones, L. y Rodríguez, M. Uso potencial de las mezclas de biodiesel de Jatropha y de Palma en Colombia. Studiositas: 5(3), 75 - 87 (2010)

Xin, J., Imahara H. y Saka, S. Kinetics on the oxidation of biodiesel stabilized with antioxidant. Fuel: 88 (2), $282-286$ (2009).

Zuleta, E. Estudio de la estabilidad oxidativa de biodiésel de aceite de palma en contacto con materiales metálicos y poliméricos automotrices. Ingeniería y competitividad: 14 (2), 83 - 90 (2012a).

Zuleta, E., Rios, L. y Benjumea, P. Oxidative stability and cold flow behavior of palm, sacha-inchi, jatropha and castor oil biodiesel blends. Fuel Processing Technology: 102 (0), 96 - 101 (2012b). 